\title{
Development of linguistic personality under the influence of social and cultural factors of the modern society
}

\author{
O.A. Pobegaylov \\ Cand. of Sc.( Economic) \\ Don State Technical University \\ Rostov-on-Don,Russia \\ oc41@bk.ru
}

\author{
Y.P, Khoroshevskaya \\ Cand. of Sc.( Philology) \\ South Federal University \\ georgy-2583@yandex.ru
}

\author{
L.P. Novikova \\ Don State Technical University \\ Rostov-on-Don,Russia \\ dgtu.kafedraos@yandex.ru
}

\begin{abstract}
The article studies the behavior of the linguistic personality in the modern information space. The problem of linguocultural interaction seems interesting for philosophy, philology and linguodidactics. But we must agree that language and culture form a dialectical unity (although contradictory) and the relationship between language and culture is characterized by today's scientists as interpenetration. The existence of man in modern society imposes a visible imprint on his personality as a bearer of language. The article studies the acute problem of modern society - the development of the linguistic personality of a person in the conditions of modern society.An analysis of the impact factors on the individual at the linguistic level is carried out. A level classification of these factors is given..
\end{abstract}

Keywords - language personality, information culture, theory of linguistic

\section{INTRODUCTION}

The culture of speech, which is a conditional system of interacting different-level elements of a common language, is an indispensable feature that determines the cultural and historical component of the epoch on a linguistic level. By studying the stylistic layers inherent in this or that historical and social type of speakers, a philologist is likened to an archaeologist who studies the past through cultural layers. Research of the linguistic personality in different historical periods allows us to study not only the practical use of language facilities by a native speaker, but also the conception of the language culture essence in a certain period of time. Comprehending language as a phenomenon of nationwide and world culture, we examine it in indissoluble connection with the social and cultural phenomena of the epoch as one sociocultural process. Research of the modification of the sociocultural component of the linguistic personality during of the historical process is among the priority purposes of linguistics, as, for example, V.I. Karasik says about it [1]. For achievement this goal it is necessary to solve the following tasks: to classify linguistic personalities by typological features; identify the main linguistic features inherent by linguistic personality, which are appear during of speech practice; to analyze degree of their changes in the dynamic development of the linguistic personality in the historical process.

\section{INFORMATIONAL BEHAVIOR OF LINGUISTIC PERSONALITY}

The study of the linguistic personality involves a solution of the problems of classifying linguistic personalities according to certain characteristics, which makes it possible to systematize the main features of the native speaker, manifested both in synchrony and in diachrony. Most scientists (for example, V.I. Karasik, Y.N. Karaulov, L.M. Kolesnikova, etc.) consider the linguistic personality within the framework of the integrative approach as a multidimensional phenomenon, determined by various factors. The most important constitutive principle that allows and actively implements to integrate various components of the linguistic personality is a communicative strategy. It is obvious that the principles of communicative strategy are immanent for any speaker, since the earliest study of the language as an ability to realize speech activity presupposes the mastery of one of the communicative strategies. In the process of personal development, communication strategies are being improved, while social factors play an important role: professionalization, socialization, etc. Communicative strategy determines the choice of linguistic facilities by the communicator and the realization such image of the speech culture, which is most justified in certain communicative situation[2-6].

From this point of view, we can distinguish the following types of linguistic personality:

- authoritative linguistic personality, the main features of which are: communication control approach, authoritative, unilateral way of conducting discourse $[58 ; 62]$;

- a marginal linguistic personality using the most simplified communication system, a denominated language consisting of dialectisms, jargonisms, argotisms and oral forms in their mixing [6,7];

- a literary personality, i.e., the personality of an individual who actually existed, in conjunction with a greater or lesser distraction from reality, implemented in the text. Under the literary personality are understood both the author identity, and the images of characters embodied by him into the text [9-12]; 
- Co-operative linguistic personality, who chooses during of natural communication cooperative (non-conflict) strategies, tactics and arrange them ways of communication [11];

- dialectal linguistic personality, i.e., the linguistic personality of a linguistic system carrier, the use of which is territorially and culturally limited $[1 ; 12 ; 13-16]$;

- the image of the rhetorician, a rhetorical personality, considered as a rhetorical category, determining the linguistic qualities of the speaker in the process of implementation in public discourse [7];

- Ethnocultural (ethnosemantic, national) linguistic personality as the focus of the national mentality - specific worldview attitudes, value priorities and behavioral reactions, enshrined in the language of the ethnos $[12 ; 13 ; 18]$;

- an emotional linguistic personality, i.e., a linguistic personality, considered from the point of view of the ability to express your own emotions verbally $[19 ; 20]$;

- gender linguistic personality, based on the specifics of verbal activity which "is a gender type personality (masculine, feminine, androgynous)" [7, c. 91];

- the dictionary person as a generalized "carrier of representations, stereotypes and norms fixed in the meanings of words interpreted in dictionaries" $[4 ; 6]$;

- a historical linguistic personality, i.e., "a linguistic personality belonging to another historical epoch" [4].

Communication between different persons in the native language takes place through significant integral semantic segments that have internal logic, conceptuality and indivisibility. Thus, when analyzing the linguistic representation of an individual, it is necessary to consider no separate minimal elements of the language used by the personality, but the whole system and the entire array of phraseology and syntax used by the individual. This becomes particularly evident when we examine the dynamics of the linguistic personality development, since even formally minor changes in the lexical structure of the vocabulary are in fact global shifts in terms of communication and the actual use of the vocabulary. T.M. Dridze notes that native speakers knowable know the precedent texts that serve as sources of cultural and national interpretation of phraseological units, and, more broadly, the entire composition of produced texts. Identification of stable elements of the language is possible only on sufficiently represented arrays of ideographic fields (such as "face properties", "feelings", "intellectual abilities and human states", "behavior", etc.) [2,8].

\section{EVALUATION METHODS AND SOURCES OF INFORMATION}

A specific feature of the realization of the linguistic personality is the using of everyday speech in the process of constant communication and the conscious selection of means for speeches in special cases. It should be take into consideration that the genre specification for the majority of native speakers is largely a formality and the selection of funds in advance of the prepared text is conducted from the same generalized basis of everyday speech. In the process of communication, the linguistic personality operates with limited tools of vocabulary, phraseology, grammar (in written speech) syntax and stylistics with the following characteristics:

1. Spontaneity is a variable category that is realized depending on the situation in which the linguistic personality that produces speech is found. It should be noted that spontaneity can be realized in the mind of the perceiving personality, when even a specially organized text is perceived differently than it is planned, because the recipient is not ready for communication. But this phenomenon is to a large extent in the field of personality psychology and is conditioned primarily by the factors of perception, thinking, individual psychophysiological processes, the emotional state of the individual, rather than purely linguistic or recitative $[15$, c. 11 ; 55].

2. Monologue and polylogue - depending on the type of communication, a monologue, a dialogue or a polylogue is carried out - a text is formed and speech means of expressing the speaker's thoughts are selected. At the same time, the monologue, dialogue and polylogue influence on the degree of compression of the utterance, and non-verbal aspects - on the speed and length of the speech act and the justification of selection of speech resources. The situations of a monologue and a polylogue initially presuppose a certain scheme of interaction between a linguistic personality and determine the plan for expressing a given conceptual word by means of linguistic features.

3. Hesitation. The filling of the pauses in the speech stream is more characteristic of live speech, however, in modern speech discourse in the virtual space of the Internet and in mass media oriented to the presentation of direct, unformalized speech, a similar phenomenon is observed in writing. For example, the record of S. Buntman's and A. Kuznetsov's interview, presented on the website of the radio station Ekho Moskvy, contains the reproduction of redundant speech elements that are important for preserving the conversation style, but not for conveying the information component of the conversation: "This, so to speak, on the one hand led to the idea that one should not do, so to speak, such a semi-closed or completely closed process, one has to baboon " [65].

4. Accentuation of the person (anthropocentricity of everyday speech). Constant, often unjustified emphasis on the importance of the speaker's personality in the process of implementing the communicative act. Personality accentuation is realized by various methods, such as: deliberate use of personal pronouns, including anthropocentric combinations ("I / me-expressions"), for example, "as I call it," as I said, "" as I defined "," I have been told / done / determined / named "; repetitions of aphoristic statements containing the speaker's point of view; wide autocytacy ("repeatedly repeated," "repeatedly said," etc.); an attack on the opponent's personality, which amounts to demonstrating a social, cultural, intellectual or other difference in favor of the speaker; an appeal to the personal merits of the speaker as the leading argument.

The phenomenon of personal accentuation in a communicative act in the text as a whole has become most widespread in journalistic texts, as well as in various discussions in Internet conferences. It should be noted that 
often self-realization of a person in public discourse is reduced to self-assertion by means of certain texts, where the personality of the speaker is the main subject of consideration, even if a different problem was stated [9].

5. Epatage. Shocking of interlocutors or addressees of a rhetorical performance is one of the common methods used by representatives of various social groups in public discourse. It should be noted that outrage in the process of speech practice plays different functional roles, becoming a means of expressiveness, emotion of speech or text, then an element of stylization, then a means of manipulating the consciousness of the addressee, or a form of the author's presentation (for example, to emphasize belonging to a particular social or political group). It should be noted that at the present time there are statements from representatives of various arts and social movements that lead to the outrageous leading role in creativity, believing that shocking an addressee is a priority incentive (for example, to ward off a person from smoking, he is shown appropriate images and texts that cause rejection, shocking the potential consumer). Also in a number of cases there are utterances containing a statement in the priority of shocking over any other meaningful elements of the text, including those formed in the process of bilateral or multilateral communication [20]. This point of view mainly prevails among the people who are the authors of the so-called "web-literature" works, including journalistic works.

The game in unwarranted, in evasion of the interlocutors by tabooed speech means is quite common in public discourse and even becomes the calling card of some mass communication means that attract potential consumers with texts of "forbidden" content, playing on the quasi-expansion of the individual's possibilities in the process of direct or indirect communication.

6. Compression. The abbreviations adopted in speech practice are a huge class of various phenomena that allow the linguistic personality not only to reduce the volume of the utterance, but also to increase its expressiveness, and also, in some cases, express its attitude. Compression can be represented in the form of a classification category in which different types of sentence reduction are divided into types:

- Abbreviation. The formation of abbreviations of various types is not only necessary for the implementation of communication conditions of limited technical means of volume, but also the possibility of expressing thoughts with a certain stylistic coloring or additional content that is not invested in the original concept. On this fact, for example, the whole work of Arkady Gaidar "R.V.S.N." was built, in which the abbreviation acquires a special sacral meaning for the main character. This technique is widely used in modern public discourse, when using the abbreviations and words formed from abbreviations (for example, the KGB is a KGBshnik, etc.), the speaker's attitude to the text is transmitted. The abbreviation in the perception of the linguistic personality turns out to be something more than a shortening of the word for its transmission over a limited information channel. For example: "We should not perceive journalists as KGBists conducting interrogation" [3]. Here there is a semantic game in which two words are formed by the same postfix, but are opposed both in meaning and stylistically.

- Forms of graphic compression. Abbreviations through truncation of words with the addition of a punctuation mark are in many respects similar to abbreviations, but, as a rule, do not serve to express personal judgment or emotions of the speaker.

- Contamination. The formation of contaminated words serves primarily as a means of expressiveness, a reduction in volume, with an increase in the significance of the word and a simultaneous marking of the latter. Contamination in speech practice always has an occasional nature and reflects the perception of the speaker of the lexical base of the language.

- Metonymy (metonymic reduction). Along with the ellipse, metonymy provides marking of the expression using standard lexical means. It should be noted that metonymy, along with metaphor, has always been one of the main means of expressing personal concepts and one of the means most fully characterizing the specificity of the linguistic personality.

- Ellipse. Quite common in colloquial speech, but in the written forms of public discourse are relatively rare.

- Semantic condensates. Formation of a new word by splicing parts of stable expressions into a single root word. Semantic condensates are similar to contaminated formations, but do not have such a pronounced occasional form that do not express the speaker's relationship to the subject, except for an emphatically informal style of speech. Compression plays an important role in determining the properties of the speaker's personality, his perception of the language, the communicative situation, the relation to the interlocutor and the reality.

7. Speech etiquette. Etiquette forms of interaction differ significantly in the historical aspect, each epoch presented its criteria for speech etiquette. Nevertheless, many concepts have acquired the character of universals, entrenched in national culture, and are preserved in speech, being modified in the process of changes in the sociocultural environment. A vivid example of the modification of etiquette forms of interaction is the modification of the appeal "comrade". The word from everyday vocabulary of the national language in the end of the XIX - the beginning of the XX century was transformed into treatment with the expressed ideological characteristic, then, in the territory of the USSR, it turned into a stylistically neutral everyday appeal to any person, then, at the turn of the XX-XXI century, and became a marker of certain sociocultural relations in the communicative process, acquiring in a concrete context a historically not peculiar ironic, positive or negative meaning. Etiquette forms of interaction can be divided into two major groups: A) non-verbal - the most common in Internet communication and SMS-correspondence, where the verbal and tone equivalent is transmitted by conventional symbols, as well as the color and size of letters (red color is the prerogative of the resource administration, allocation bold - the strengthening of the meaning of the word, intonation analogue of the phrase stress, writing in capital letters - shouting, talking in elevated tones). B) verbal. In addition, there are combinations of verbal and non-verbal means, allowing you to convey the emotional state of the speaker on the letter. For example, the use of the "closed parenthesis" on the letter after 
the statement means a smile, an ironic attitude of the writer to the said. Etiquette speech facilities can be classified as follows:

- Appeals. The etiquette forms of addressing to the interlocutor are often expressed, modified by the speakers depending on the role played by the image of the interlocutor in communicative interaction, while the appeal acquires vivid, imaginative features, drawing nearer to the means of representativeness: "I go to him, I show bruises:" Vanya, you're a stupid ram! What are you doing? ")» [8]. The appeals can be classified according to the method of education in the form of appeals, occasionalisms, the treatment of exoticism, treatmentmetaphors, appeals by profession, social, intellectual and other status, appeals by kinship, personal sympathies, disagreements, appeals for a role in virtual interaction.

- Diminutives. A specific form of access, which is logical to consider separately. In contrast to additional value added speaking in the usual treatment diminutives originally expressive transmit tagged value that becomes clear regardless of context.

- Euphemisms. Traditionally, euphemism is understood as the replacement of an undesirable token or expression by a neutral means, but an analysis of the historical sources and materials of modern speech practice shows that this technique is of far greater importance in public discourse than is traditionally represented. Euphemization is a full-fledged tool for modifying text and building communication in a way that the speaker wants. Moreover, the selection of euphemisms is an instrument of influence on the audience, which allows to form not only the image of the speaker as a person using certain etiquette forms to represent the problem in the proper perspective, but also the image of the audience to whom it is addressed. For example, the textbook phrase "not theft, but unauthorized selection" (V.S Chernomyrdin) not only replaces the undesirable word in the diplomatic (professional) context, but also creates a different image of the mediated subject of the speech of the author of the statement. On the other hand, there is also an inverse de-efeminization process, for example: "And another component of the losses, which the director of the housing service modestly kept silent: unauthorized selection of electricity, or simply stealing" (Official website of the newspaper "My City Severodonetsk" dated December 8, 2013 17:59). In this case, the etiquette form is deliberately replaced by a reduced form. Euphemisms largely determine the speaker's practice and communication experience, the ability to analyze and take into account the interests and qualities of the audience, the degree of his language proficiency and the ability to create various options at the lexical, syntactic and stylistic levels of the language.

8. Word creation. Word creation - is a huge reservoir of vocabulary, born spontaneously, it displays individualpersonality traits of a native speaker, his perception of language and communicative situation in which it is implemented. Word creation is not a necessary element of interpersonal and group interaction, but it reflects the need for self-realization of the speaker, primarily in creating a unique word that fully reflects both the semantic and stylistic aspects of the definition or concept.
9. Visual and expressive means. The use of tropes and figures of speech is the most studied aspect of the realization of the linguistic personality in practical activity. Therefore, the authors do not consider it necessary to specifically focus on this phenomenon, but only note that the graphic and expressive means are used by all native speakers in public discourse and serve as one of the main characterizing values of the linguistic personality.

10. Citation. The use of quotations in speech communication is a rather old and widespread phenomenon. Being more in the center of attention anthropology and epistemology, citation, however, is also one of the constituent characteristics of the linguistic personality. The quotation, from the point of view of linguistics, shows the reader's experience, the understanding of the text and language as a material embodying the meaning, serves as a means of a brief and meaningful expression of thought, and, in some cases, a means to enhance the expressive or visual qualities of the text. Functionally, a quote can come close to traditional means of language, for example, expressive means, while remaining an integral independent statement. The appropriateness, justifiability and accuracy of citations determine the speaker's ability to produce stylistically correct text. On the other hand, citation is one way to prove one's point of view $[16,19]$. In this aspect, a phenomenon is manifested, such as quasi-citation attribution to a known person of statements that do not belong to him. Quasi-quoting is a fairly common technique, while a false quote, as a rule, is an aphoristic utterance, the authorship of which, most often, belongs to the speaker himself. The need for quasi-citation shows that for the linguistic personality it is important not only the content of the text, but also the authority of the speaker, that is, the linguistic personality turns out to be a self-valuable phenomenon existing outside historical parallels, and the degree of its importance for other communicators directly depends on their life position, cultural preferences, education, social role and those characteristics that they choose as the determining factors in the evaluation of the linguistic personality of the communication partner.

Since the 90-ies of the twentieth century, the volume of constantly produced textual information has been growing all the time, regardless of the way a linguistic personality works. People of all social groups and professions were involved in the global exchange of textual information, now equal in volume to oral speech.

After the information revolution, the pinnacle of which was the creation of the Internet, information technologies define the entire sphere of human activity, regulate it. And at the present time the main organizational and managerial role has shifted to a text of different types and content, since at the present time the exchange of textual information, including regulatory and organizational nature, has reached an absolute maximum, significantly pushing the interaction in the telephone, telegraph and other modes. The ability to instantly transmit text messages of unlimited volume, the formation of text in real time, the cumulative work on the general text of remote users, the exchange of textual information with the modeling of various socio-psychological situations, previously possible only with direct communication - all this is decisive in modern practice rights. In fact, it is the professional text of various 
types that turns out to be the main product and tool of the present, with an intrinsic unique value.

As the experience of recent events, the Internet space has become an important segment of the interaction between the individual and society. Donald Trump victory in the presidential election, according to some experts, is largely due to skillful self-presentation and communication Trump with society through the Internet. The phenomenon of Internet communication represents significant interest for the studying of the linguistic personality evolution, since, in comparison with the evolution of the linguistic personality and the historical linguistic personality in other communicative environments, in the Internet environment, the linguistic personality appears in a specific virtual space and has features of the speech appearance different from other forms of linguistic personality.

The problems of Internet communication in recent decades have been studied extensively by linguists. But a significant amount and variety of forms of information in networks and communications between users and the speed of evolution of the Internet environment leaves a wide field for study.

Nevertheless, the volume studied at the present time allows us to draw a number of conclusions about the fundamental features of the linguistic personality representation in the Internet space.

As noted by A.V. Belozertsev, L.K Churina and others, virtual reality has a spatial and temporal extension, signs of a material-tangible world, transposed into the consciousness of communication participants, i.e. imagined by him under the influence of a specific environment, which is formed in the process of communication by the communicants themselves. It is important to note a significant difference that was not observed by the first researchers of the Internet environment: the virtual space in many respects differs from the public language space, formed in the media and other forms of group social interaction.

The virtual world has important features that affect the speech activity of a person who communicates actively in it.

The linguistic personality in the virtual space has the following characteristics:

- expressed imagery, creating an active mask (using of nicknames, aliases);

- striving for the promotion of the interlocutor and selfpromotion, programming for certain actions of a positive and negative nature by linguistic means (for example, a phenomenon such as "death groups" in social networks that provoke suicide).

- striving for constant enlightenment, preaching any ideas, creation various texts, including ritual-religious;

- the demand for communication with other individuals in a virtual network;

- at the same time on the ideological level linguistic identity in the Internet environment tends to create an image of an ideal world, represents the existing reality in both synchronic and diachronic aspects in the desired manner, sometimes deliberately distorting it.

The competition of linguistic personalities of public figures of different religious, political and other kinds, thus, predetermined the use of emotionality as a tool of influence on the audience. In the modern Internet environment there is an acute struggle for emotional dominant. The authors of publications and messages in Internet conferences build communication as an exchange of emotions, the creation of a single verbalized emotional space, where the actual information component is minimized, and the interaction is only due to emotional empathy or rivalry. Numerous conflicts in blogs and forums have a pronounced emotive nature and serve as an additional means of organizing the authors of likeminded and statements of the point of view. This purpose is served by the phenomena of "trolling", "Internet bots" specialized techniques aimed at artificial creation of conflict situations and maintaining the intensity of the emotion with the aim of creating a sustainable, desirable, a priori associative perception of the image of one or another public figure and put forward their ideas.

The specificity of the agitator in the modern Internet is to create the desired emotional image, achieve emotional contact, while the rational and the actual side of the issue is not important. For example, the stated topic of an Internet conference may concern the history of literature, while the actual case may be defined as the achievement of "emotional kinship" of the participants.

\section{CONCLUSION}

Modern society is fully integrated into a virtual environment, where the main element of interaction is text. Regardless of whether this phenomenon turns out to be a permanent civilizational feature, fixed like the epistolary forms of interaction, for several centuries, or it will be replaced by a new phenomenon within one or two generations, this phenomenon has a significant impact on today's reality and is included in all spheres human interaction, is a full-fledged element of world culture.

The linguistic personality represents a continuous dynamic subject with a pronounced functionality, the ability to selforganize, self-learn and improve in different conditions. The texts generated as a result of the activity of the linguistic personality throughout the whole period under study are constantly becoming more complex and becoming more and more important, becoming almost the main means of exchanging interpersonal and group communication by the end of the first decade of the $21 \mathrm{st}$ century. In the process of development, there is an indissoluble connection between the society, the national and world culture and language, manifested in the speech representation of the individual's speech-activity and in the text produced by him.

\section{REFERENCES}

[1] Karasik V.I. Aspects of the language personality // Problems of speech communication. - Saratov, 2003. - P. 96-106. 
[2] Abulkhanova-Slavskaya K.A. Personal types of thinking // Cognitive Psychology: Materials of the Soviet-Finnish Symposium. - Moscow: Nauka, 1986. - P. 164-173.

[3] Guts E.N. Profanity in the speech of modern urban adolescents: in the light of the concept of language personality: the author's abstract. dis. ... cand. philol. sciences. - Barnaul, 1995. - 210 with.

[4] Arutyunov S.A. Ethnic processes and language // Introduction to ethnolinguistics: The course of lectures and reader. - St. Petersburg .: Publishing house S.-Petersburg. University, 2001. - P. 164-184.

[5] Vorozhbitova A.A. Linguoritoric paradigm: theoretical and applied aspects. - Krasnodar, 2000. - 110 with.

[6] Bogin GI The model of the language personality in its relation to the varieties of the text: dis. ... Dr. Philol. Sciences / G.I. The goddess. - L., 1984. - $312 \mathrm{p}$

[7] Vinokur TG Speaker and listener. Variants of speech behavior. - M., 2005. - $80 \mathrm{p}$.

[8] Guboglo M.N. On the influence of resettlement on language processes // Soviet ethnography. - 1969. - No. 5. - P. 76-89.

[9] Vorkachev S.G. Linguoculturology, the linguistic personality, the concept: the formation of the anthropocentric paradigm in linguistics // Philological sciences. - 2001. - No. 1. - P. 64-72.

[10] Golubeva I.V. The experience of creating a collective speech portrait (on the material of the expressive syntax of memoir prose): the author's abstract. dis. ... Dr. Philol. Sciences / I.V. Golubeva. - Krasnodar: KubSU, 2002. -45 p.
[11] The width of E.V. The concept of "personality" in the context of journalistic and linguocultural research Bulletin of St. Petersburg University. Series 8. Management. 2008. № 2. P. 325.

[12] Usheriev Yu. D.. Social linguistics. - M., 1976. - 170 p.

[13] Shirina E.V, Myasishchev G.I. The role of effective linguistic communication in the management of the company. The Engineering Bulletin of the Don. 2017. 47. No. 4 (47). P. 121.

[14] Karasik V.I. Cultural dominants in the language // Language personality: cultural concepts. - Volgograd, 1996. - P. 3-16.

[15] Karasik V.I. Language identity and language categories // Language personality: problems of meaning and meaning: Sat. sci. tr. - Volgograd: The Change, 1994. - P. 25-35.

[16] Shirina E, Gaybarian O., Myasischev G Effective management of construction company in terms of linguistic communication IOP Conf. Series: Earth and Environmental Science 90 (2017) 012077

[17] Karaulov Yu.N. Russian language personality and the tasks of its study // Language and Personality. - Moscow: Nauka, 1989. - P. 3-178.

[18] Karaulov Yu.N. Russian language and language. - M., 1987. - 371 p.

[19] Shirina E.V. Word - concept - concept (the ideological world of civil personality in the evaluations of publicists-democrats of the 13th century) Bulletin of the Adyghe State University. Series 2: Philology and Art History. 2009. № 2. P. 53-59.

[20] Dmitriuk N.V. Associative behavior and national and cultural specifics // Text as a tool for communication. - M., 1983. - P. 81-86. 